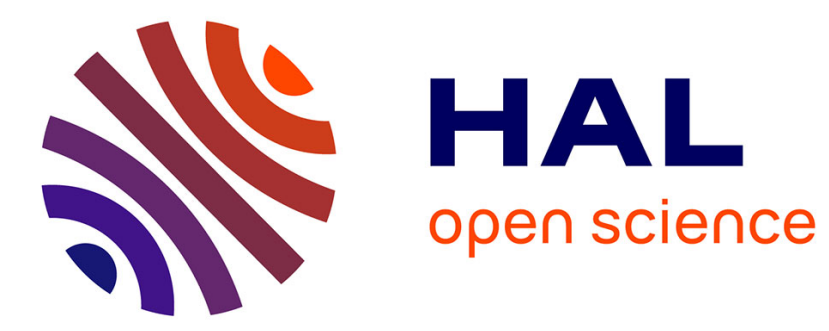

\title{
Au-delà des métamorphoses du corps : la chair du psychique \\ François Villa
}

\section{To cite this version:}

François Villa. Au-delà des métamorphoses du corps: la chair du psychique. Corps \& psychisme: recherches en psychanalyse et sciences , 2008, 50 (2), pp.67 - 91. 10.3917/cpsy.050.0067 . hal01496263

\section{HAL Id: hal-01496263 \\ https://hal.science/hal-01496263}

Submitted on 27 Mar 2017

HAL is a multi-disciplinary open access archive for the deposit and dissemination of scientific research documents, whether they are published or not. The documents may come from teaching and research institutions in France or abroad, or from public or private research centers.
L'archive ouverte pluridisciplinaire HAL, est destinée au dépôt et à la diffusion de documents scientifiques de niveau recherche, publiés ou non, émanant des établissements d'enseignement et de recherche français ou étrangers, des laboratoires publics ou privés. 


\section{AU-DELÀ DES MÉTAMORPHOSES DU CORPS : LA CHAIR DU PSYCHIQUE}

François Villa

L'Esprit du temps | «Champ psychosomatique »

2008/2 n $50 \mid$ pages 67 à 91

ISSN 1266-5371

ISBN 9782847951271

Article disponible en ligne à l'adresse :

http://www.cairn.info/revue-champ-psychosomatique-2008-2-page-67.htm

\section{Pour citer cet article :}

François Villa, «Au-delà des métamorphoses du corps : la chair du psychique 》, Champ psychosomatique 2008/2 ( $\mathrm{n}^{\circ} 50$ ), p. 67-91.

DOI 10.3917/cpsy.050.0067

Distribution électronique Cairn.info pour L'Esprit du temps.

(C) L'Esprit du temps. Tous droits réservés pour tous pays.

La reproduction ou représentation de cet article, notamment par photocopie, n'est autorisée que dans les limites des conditions générales d'utilisation du site ou, le cas échéant, des conditions générales de la licence souscrite par votre établissement. Toute autre reproduction ou représentation, en tout ou partie, sous quelque forme et de quelque manière que ce soit, est interdite sauf accord préalable et écrit de l'éditeur, en dehors des cas prévus par la législation en vigueur en France. Il est précisé que son stockage dans une base de données est également interdit. 


\title{
Au-delà des métamorphoses du corps : la chair du psychique
}

\author{
François Villa ${ }^{1}$.
}

'Qui pourrait en effet retourner d'un coup vers l'extérieur la doublure du temps?»

Walter Benjamin.

\section{«UN PEU DE TEMPS À L'ÉTAT PUR ».}

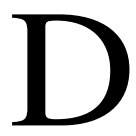
ans «Pour le portrait de Proust», Walter Benjamin écrit: "Proust est pénétré de cette vérité que les vrais drames de l'existence qui nous est destinée, nous n'avons pas le temps de les vivre. C'est cela qui nous fait vieillir. Rien d'autre. Les rides et les plis du visage sont les enregistrements des grandes passions, des vices, des savoirs qui se sont exprimés en nous - mais nous, les maîtres, nous étions absents » ${ }^{\mathbf{1}}$.

Proust réussit, dans «Le temps retrouvé », ce tour de force qui rend perceptible pour le lecteur une expérience : celle où l'instant d'un souvenir involontaire laisse vieillir le monde entier autour d'un homme. Celui-ci voit, sans pouvoir protester, dans la première glace véridique, le temps, qui d'habitude n'est pas visible, exprimé en son corps. Le souvenir involontaire défie l'oubli où le tenait le moi. Et, il réduit le monde, par la violente contraction qu'il lui fait subir, à n'être plus que l'intensité matérielle de l'image, de l'odeur, de la
1. Benjamin W., in Mythe et violence, trad. M. de Gandillac, Paris, Denoël, p. 326 .

François Villa - Psychanalyste, Maître de Conférences (HDR), membre du Centre de Recherches en Psychanalyse et Médecine (ED Recherches en Psychanalyse); Université Paris 7 - Denis Diderot. 30, bd de Strasbourg, 75010, Paris, villa@univ-paris-diderot.fr. 
2. Proust M., «Le temps retrouvé, in À la recherche du temps perdu, t. III, Paris, Ed. La Pléiade, Gallimard, 1977, p. 872.

3. Nous pensons à la réponse que Lenehan fait à O'Molloy : «Un-deces-décès-subito-prestoencore-d'une-longuemaladie-suite-et-fin... Et avec un grand avenir derrière lui», Joyce J., Ulysse, trad.. A. Morel,

Paris, livre de poche, 1965, p. 136.

4 Freud S., Résultats, idées, problèmes, trad. J. Altounian, A. Bourguignon, P. Cotet, A. Rauzy, in Résultats, idées, problèmes, $\mathrm{t}$. II, Paris, Gallimard, 1985, p. 288 . saveur qu'il a imposée à la mémoire. Le temps qui d'habitude n'est pas visible, dans cet instant, la durée d'un éclair, défie la loi inévitable qui veut qu'on ne puisse imaginer que ce qui est absent. Un bruit, une odeur, un contact, un presque-rien «fait miroiter une sensation [...] à la fois dans le passé, ce qui permettait à mon imagination de la goûter, et dans le présent où l'ébranlement affectif de mes sens par le bruit, le contact du linge, etc. avait ajouté aux rêves de l'imagination ce dont ils sont d'habitude dépourvus, l'idée d'existence $»^{2}$.

Mais, le souvenir involontaire, bien qu'il se révèle doté d'une éternelle actualité et qu'il ait ce pouvoir d'amener le monde à s'invaginer en lui, rencontre, en sa circularité-même, la limite où, au moment de sa plus forte brillance, il pâlit soudainement et nous laisse consciemment vieilli. Le souvenir involontaire, qui défait la censure du moi, est un oubli de moi. C'est la rencontre imprévisible avec soi qui provoque ce moment où l'on se voit alléger de la pesanteur de la tentation d'identité du moi, pour n'être qu'un lieu : celui d'une inquiétante et étrange familiarité. Ce lieu d'une extrême fragilité est un moment hors monde et hors conscience de pure conscience de soi. En lui, survient l'oubli de moi qui permet l'advenue non pas d'un souvenir, mais d'un signal : celui du trop tard, de ce que nous n'avons pas eu le temps de vivre, de ce qui, trop fugace, trop ténu, n'avait eu que la possibilité d'exister sans jamais venir à la conscience. L'éclair d'un instant, nous appréhendons ce que jamais nous ne saisirons : « un peu de temps à l'état pur » et, dans cette béance, apparaît la négligence du temps dont se soutient le cours d'une vie. Jamais nous ne saurons si le souvenir involontaire est un souvenir fidèle des événements qui arrivèrent dans le passé ou un souvenir que nous rattachons à ce passé. Il est néanmoins l'instant où l'homme découvre qu'il a un grand avenir derrière lui ${ }^{3}$ en percevant l'une des croisées du chemin où s'est nouée, à l'insu de sa conscience, sa vie. Celui qui consent à l'oubli de moi cesse de réprimer la mort et les morts qu'il porte en lui et peut, à partir de la chair du monde qui est sa chair, avoir " l'obscure auto-perception du royaume extérieur au moi, au ça $»^{4}$.

Selon Proust, c'est leur manque d'imagination, et non pas leur courage, qui fait que quelques uns parmi les hommes s'affrontent avec indifférence à la vieillesse comme à la mort. Ce défaut d'imagination, nous en trouvons la ressource dans l'oubli quotidien de nos métamorphoses, dans cet oubli qui 
nous fait nous tenir pour même celui que nous fûmes enfant, celui que nous étions encore hier et celui que fugitivement nous sommes. C'est l'oubli que, toujours, comme une eau qui coule, la forme en une autre s'en va 5 .

Quand un ami, que nous n'avons pas vu depuis un certain temps, s'extasie en prétendant que nous n'avons pas changé, grande est notre inclination à le croire, alors que ce dont nous devrions être certain c'est qu'il trouve que nous avons fort vieilli. Et, pourtant, faisant cette expérience, je n'en conclus que difficilement que cela me concerne tout autant. «Car, comme l'écrit Proust, nous ne voyions pas notre aspect, nos propres âges, mais chacun, comme un miroir opposé voyait celui de l'autre ${ }^{\mathbf{6}}$.

\section{L'ÉBRANLEMENT AFFECTIF : LE TEMPS DE L'AFFECT}

Immanquablement, surviennent ces instants de souvenir où se déchire le voile de l'oubli jeté sur nos métamorphoses. Nous voyons, alors, d'un visage, les états successifs ${ }^{7}$ qu'il nous faut traverser pour reconnaître, dans celui qui se tient devant nous, celui que nous connaissions autrefois. Comment penser, sans trembler, que notre propre corps nous est triplement inconnu, d'abord nous ne savons ni ce qu'il est, ni ce qu'il peut, ensuite nous méconnaissons le en devenir qui l'a fait être ce qu'il est aujourd'hui et enfin nous imaginons le moins possible ce qu'il sera demain. Nous ne voulons savoir que peu de choses de la drôle de navigation temporelle qu'il nous impose.

La survie du corps ne dépend principalement que du fait que les exécuteurs et les protecteurs de la vie ${ }^{8}$, qui œuvrent au sein de chacune des cellules qui le composent, travaillent, d'une façon telle que ni son organisation, ni le schéma corporel, ni les images inconscientes et conscientes du corps ne sont brutalement remises en question. Tant que je peux entretenir l'illusion d'une ressemblance à moi-même, peu me chaut que, quotidiennement, surviennent le suicide de milliers de cellules et la naissance de milliers d'autres. Que ce soit au prix de la mort qui œuvre, dans le for intime, que se sculpte la forme du vivant m'indiffère tant que je reconnais cette forme humaine que ma passion anthropomorphique me fait prendre pour le visage humain.
5. Je cite Ronsard dans son Hymne de la mort : «Ce qui fut se refait; tout coule comme une eau, / Et rien dessous le Cielne se voit de nouveau;/ Mais la forme se change en une autre nouvelle /Et ce changement là, Vivre, au monde s'appelle, /Et Mourir quand la forme en une autre s'en va».

6. Proust M., Le temps retrouvé, in À la recherche du temps perdu, t. III, Paris, Ed. La Pléiade, Gallimard, 1977, p. 930.

7. Ibid., p. 922.

8. Ameisen J.-C., La sculpture du vivant, Paris, points sciences, Seuil, 2003. 
Mais, avec le temps, la déchirure du voile persiste et, malgré notre Dur Désir de durer (P. Eluard), nous ne pouvons plus méconnaître les profonds sillons que le temps a creusés sur le corps. Et, nous nous surprenons, plus souvent que de coutume, à épier notre corps, nous avons alors pris conscience du vieillissement. Le plus pénible quand on vieillit, n'est-ce pas de se sentir, assez souvent, toujours jeune? Une patiente disait fort à propos : «Je me sens plus jeune que jamais, mais mes os, pas ». D'une part, cette discordance dans la perception ouvre sur la béance qui existe entre tout ce qui encore aspire à naître et l'obscure perception que le temps manquera pour le mettre en monde. D' autre part, en elle, se fait entendre tout ce qui n'en finit pas de mourir en nous: les désirs infantiles, les souhaits inaccomplis, les affronts non lavés, les blessures que le temps n'a pas cicatrisées et tous ces morts qui, de n'avoir pas reçu sépulture, continuent de vieillir en nous - réclamant que soient accomplis les rituels d'inhumation qui leur sont dus.

C'est dans la pulsation de ce matériau accumulé auquel tout traitement psychique a été refusé que prend racine notre perception fugace du temps. Ce qui est négligé, lorsque n'est pas consenti le travail psychique exigé par les nécessités de la vie, c'est le temps de l'affect, de son développement, de son miroitement dans une sensation que la conscience accueille au point que puissent alors séjourner, en elle, les pensées qui s'y rattachent. Les traces mnésiques, qui subsistent après que les choses soient passées, après leur destruction, après les pertes, nous hantent de leur espoir qu' existe à nouveau ce dont elles sont le vestige. Quand elles rencontrent, par hasard, la chance, de redevenir effectivement actuelles, que leur offre la similitude d'une odeur, d'une saveur ou d'un contact ou la ressemblance d'un visage, d'une situation, elles s'en emparent. Alors, se produit cet ébranlement affectif par où se distinguent le passé qui n'est pas passé, du présent dont l'inactualité nous échappe encore. La pulsation de ce qui persiste vivace en nous et qui, sans fléchir, se re-présente à la conscience est le battement du temps qui nous reste invisible jusqu'à cet instant où cela entre dans la conscience en pouvant y rester: s'anime alors en nous l'édifice immense de la mémoire de ce dont nous n'avions nuls souvenirs.

Cet ébranlement est aussi, toujours, un vacillement narcissique parce que nous ne ressemblons ni à ce que nous imaginions être, ni à ce que, jadis, nous avions espéré devenir plus 
tard. Cette dissemblance nous révèle, non seulement, tout à ce à quoi, malgré les modifications autoplastiques que le psychique a pu accomplir, nous n'avons pas pu donner corps, mais aussi, tout ce qui s'est vu refouler sous et dans la construction psychique du corps et qui demande, à nouveau, d'être pris en considération. Malgré, l'aiguillon qu'est l'idéal du moi, le moi est toujours loin d'être la réinstauration du moi idéal originaire.

Cet ébranlement est également le moment où se manifeste l'activité des pulsions. C'est, d'ailleurs, leur énergie cinétique qui est la cause de l'ébranlement. Une fois encore, face à ces pulsions dont la source est le soma, va se révéler l'infantilisme persistant de l'être humain. Par infantilisme, j'entends, bien sûr, l'état d'un individu qui présente encore à l'âge adulte des caractères physiques ou psychiques propres à l'enfance. Nous le savons, pour Freud, cet infantilisme est ce qui caractérise, tout au long de sa vie, le rapport de l'homme à la pulsion. L'infantile dont parle la psychanalyse est le résultat de l'impuissance dans laquelle nous plonge notre infantilisme. Dans la pulsion, quelque chose reste psychiquement, toujours et en même temps, en attente de traitement et intraitable, ce reste constitue l'infantile freudien.

\section{LA SAILLANCE : LE SOMATIQUE VA À LA RENCONTRE DU PSYCHIQUE}

Essayons de distinguer les différents éléments qui y font incidence dans cet instant du souvenir involontaire. Ce sont les restes de la vie ${ }^{9}$ passée et présente qui viennent à la rencontre du travail psychique; d'une part, les restes de quelque chose qui est en train de nous arriver et, d'autre part, les restes de ce qui nous arriva sans trouver lieu psychique. Dans ce qui nous arrive présentement, notre attention, bien plus guidée, que nous ne le percevrons jamais, par l'attente de l'infantile, cherche le détail par où il pourra accéder à son actualisation dans la mémoire. L'élément, ainsi prélevé, se voit surinvesti par ce qui, du fond de notre histoire, est venu s'y loger, il devient une saillance qui attire une autre attention, celle que contrôle la conscience. Celle-ci peut, si ce qui se présente lui est insupportable ou lui paraît inconciliable avec ses credo, lui refuser accès. Mais, si le conflit qu'elle éprouve lui paraît
9. Je reprends cette expression reste de la journée et de la vie à Ferenczi, c'est l'autre nom qu'il propose pour désigner les restes diurnes qui participent de la formation de tout rêve. Cf. Ferenczi S., Réflexions surle traumatisme, [(19201922), publication posthume 1934), trad..Coq Héron, in Euvres complètes, Psychanalyse 4, Paris, Payot, 1982,p. 141 et, aussi, Fédida P., (1985), Restes diurnes. Restes de vie, in Crise et contretransfert, Paris, PUF, 1192; Villa F. (1989), Les étrangers du jour dans l'intimité de la nuit, in Nouvelle Revue de Psychanalyse, 40, Paris, Gallimard, 127-146. 
10. Le mouvement décrit renvoie à une poussée dénuée, a priori, de bienveillance et de complaisance et, à l'égard de laquelle, il est peu probable que le moi soit bienveillant, bien que, peut-être, par nécessité, il se verra obligé de faire preuve d'une certaine complaisance envers lui. Freud S., [1925, (1926)], Inhibition, symptôme et angoisse, trad. J. Doron, R. Doron, in Euvres complètes, psychanalyse, $t$. XVII, Paris, PUF, 1992, p. 206-207. surmontable par et pour la pensée, elle laissera s'accomplir le devenir conscient par où se figurent les choses jusque-là inconscientes. Les restes de la vie présente viennent à la rencontre des restes de la vie passée qui allaient eux-mêmes vers la rencontre espérée de quelque chose qui les ferait advenir dans une forme admissible par la conscience. De cette saillie des restes du présent et des restes du passé, naît un accroissement de perception endopsychique-le plus souvent, il n'est qu'éphémère, mais parfois nous savons garder quelque chose de cette nouvelle acuité.

Pour essayer de rendre compte du chemin par lequel une issue dans le domaine du corps est donnée aux processus psychiques inconscients, Freud introduit la notion de «somatisches Entgegenkommen ». La leçon française de la psychanalyse nous a accoutumés à traduire cette formule par «complaisance somatique ». Cet «Entgegenkommen » désigne l'un des ressorts de la conversion hystérique, le mécanisme par où s'accomplit un saut du psychique dans le somatique. Sa traduction par complaisance est exacte, mais elle fait perdre toute la dynamique que comporte ce mot en allemand. «Aller ou venir à la rencontre de...» serait une manière de traduire «entgegenkommen». Quand un lieu du corps se fait saillant et vient à la rencontre du psychique, celui s'en empare et, par son travail, il dispute cette partie du corps à la fonction moïque, qui s'y accomplissait ${ }^{10}$. La zone corporelle devient alors le représentant, aussi, de l'action pulsionnelle et, parfois, elle l'est de manière tellement prédominante qu'elle en subit une restriction fonctionnelle: inhibition de sa fonction mö̈que par re-signification sexuelle. Ce qui s'accomplit est un double refoulement: le refoulement d'un conflit psychique par transposition de celui-ci dans un symptôme somatique ne s'accomplit qu'au prix d'un certain refoulement psychique de la fonction organique de la partie du corps concernée. Nous citerons un passage de Freud où apparaît nettement ce mouvement de «aller ou venir à la rencontre de $\gg$ :

«[...] les symptômes de l'hystérie sont-ils d'origine psychique ou somatique ou, si l'on opte pour le premier cas, sont-ils nécessairement tous psychiquement déterminés? [...] Le véritable état des choses ne se laisse pas enfermer dans l'alternative qu'elle propose. Pour autant que je puisse le voir, tout symptôme hystérique a besoin d'un apport des deux côtés. 
Il ne peut se produire sans une certaine complaisance somatique, qui est l'œuvre d'un processus normal ou morbide dans un organe du corps ou en relation avec lui. Il ne se produit pas plus d'une fois - alors que la capacité de se répéter fait partie du caractère morbide du symptôme hystérique - s'il n'a pas une signification psychique, un sens. Ce sens le symptôme hystérique ne l'apporte pas avec lui, il lui est conféré, il a été pour ainsi dire soudé à lui, et il peut dans chaque cas être diffèrent, selon la nature des pensées réprimées qui luttent pour $s^{\prime}$ exprimer ${ }^{11}$.

Nous le voyons, le psychique ne crée pas ex nihilo un symptôme somatique. Quelque chose préexiste dans telle ou telle partie du corps, qui peut être tout aussi bien processus normal que pathologique. Ce quelque chose vient à la rencontre du psychique qui soude alors ce qu'il veut refouler et cette partie du corps, tel est le point d'ancrage de la conversion. Nous connaissons la métaphore : il faut un grain de sable qui est la saillance d'une partie du corps - effet d'un certain processus non psychique - pour qu' autour de lui se construise par le dépôt de couches successives - processus de surdétermination psychique - la perle que constitue le symptôme psychique.

À nouveau, se manifeste la dimension brownienne du mouvement de venir à la rencontre de... Quelque chose, dans des circonstances singulières et en relation avec certaines contingences, est venue à la conscience qui, voulant s'en débarrasser, se met en mouvement vers quelque chose qui lui permettra de le faire et elle rencontre cette saillance somatique qui lui semble venir vers elle comme l'opportunité recherchée ${ }^{12}$. La levée du refoulement par l'interprétation a, le plus fréquemment, pour conséquence la cessation de la restriction fonctionnelle de l'organe. Désinvesti libidinalement, il est rendu à sa fonction moïque et voit lever le refoulement de sa fonction organique. Par contre, la dissolution des couches de nacre n'a pas pour effet la disparition du grain de sable. En cette partie du corps, que les pulsions sexuelles ne disputent plus aux pulsions du moi, peuvent subsister les processus neurophysiologiques qui en avaient fait un point de saillance somatique.

Nous avons opéré, sans l'indiquer, un glissement de l'extérieur vers l'intérieur. Ce dont Proust parlait, c'est de ce qui vient vers nous de l'évènement mondain qui survient et du
11. Freud S., [1901, (1905)], Fragments d'une analyse d'hystérie, trad. F. Kahn, F. Robert, in Euvres complètes, psychanalyse, $t$. VI, Paris, PUF, 2006, p. 220221. Pour les besoins de mon argumentation, j'ai maintenu dans le texte la traduction par complaisance somatique et ai abandonné la nouvelle traduction proposée par l'équipe des O.C.: prévenance somatique. Je souligne.

12 Villa F., Les névroses mixtes : une leçon de méthode nosographique, L'évolution psychiatrique, 72-3, 2007, Elsevier, 477-487. 
prélèvement d'un élément de cet événement qu'effectue ce qui, en nous, cherche à aller vers l'extérieur pour être perceptible par la conscience et qui, pour se faire, s'accroche à un détail extérieur qui se voit octroyer un relief singulier pour et dans notre vie psychique. Dans le passage sur la question de la conversion et de la complaisance qui la rend possible, c'est d'une autre rencontre qu'il est question. Un processus somatique survient hors de toute action du psychique, il se produit un événement somatique d'où résulte une différenciation entre la partie du corps où est localisée une certaine sensation et le reste du corps. L'événement survient à l'intérieur du corps, mais il est extérieur à l'appareil psychique. Le somatique peut alors être détaillé en différentes parties, il peut être cartographié à partir des sensations qui distinguent chacune des parties d'une autre. Par cette mise en détails du somatique, pourront se construire psychiquement le corps et les images successives que nous en aurons. Le psychique prélèvera parmi ces détails, qui se sont distingués dans l'ensemble auxquels ils appartiennent et qui semblent aller vers lui, ceux qui permettront aux motions pulsionnelles d'aller vers leur accomplissement de désir.

Revenons un instant sur ce processus normal ou pathologique qui, dans un organe ou en relation avec lui, favorise le «aller à la rencontre» du corps vers le psychique et rend possible la conversion. Même, dans le processus normal, où la fonction s'accomplit sans ratés, se produisent des variations de l'amplitude des énergies qui circulent dans le corps: des tensions, sans signification psychique, s'y génèrent et peuvent solliciter l'attention. Celle-ci peut établir des similitudes, des analogies, des ressemblances entre ces diverses manifestations normales et les processus par lesquels la pulsion pourrait s'accomplir en tant que souhait accompli. Ce travail psychique peut aboutir à poser, par la pensée, une identité, une équivalence entre la partie du corps concernée et l'organe génital. À partir de là, une nouvelle fonction: sexuelle, lui est assignée. Parmi ces variations, celles liées aux transformations du corps dans le temps occupent une place évidente: aussi bien les modifications corporelles de l'adolescence que celle de la sénescence. Le processus morbide, lui, se traduit par le dysfonctionnement d'un organe ou d'une fonction qui se manifeste par une gêne somatique, une limitation ou un embarras fonctionnel, une douleur. Ici, aussi interviennent des 
processus de variation énergétique, mais s'y rajoute un autre élément: celui de la plainte. Dans l'un et l'autre de ces cas, le processus advient, au départ, sans aucune signifiance du point de vue psychique, il constitue une saillance, plus ou moins désagréable, dans et du corps qui peut en fonction de son intensité soit former une attractivité pour l'attention, soit représenter, comme élément de coexcitation, pour le psychique une effective provocation au travail. Mais, tout ce qui précède est vrai à tout âge de la vie, aussi devons-nous demander si ce processus acquiert ou non une singularité dans le cours du vieillissement.

\section{LE REFOULEMENT ORGANIQUE ET L'ÉMERGENCE DE POINTS DE SAILLANCE SOMATIQUE}

Pendant toute une partie de la vie (qui n'est pas la plus longue), le corps, en grandissant, se développe en actualisant ses potentialités. Cette période «faste» de la vie est celle où l'enfant de l'homme passe d'un sentiment originaire d'impuissance et d'une situation de dépendance absolue à l'impression, bien réelle, d'acquérir chaque jour des forces et des aptitudes nouvelles, davantage de puissance et de plus en plus d'indépendance. Aiguillonné par l'idéal du moi, le moi se construit dans l'attente croyante qu'un jour, il redeviendra le moi idéal du début et qu'il retrouvera l'autre préhistorique et inoubliable qu'il a entrevu et perdu à l'orée de sa vie. Grâce au mécanisme du refoulement normal et/ou pathologique, il peut grandement ignorer le prix qu'il a eu à payer pour acquérir toutes ces dispositions nouvelles. Parmi ces refoulements, il en est un particulièrement important: le refoulement organique.

Ayant déjà eu l'occasion, dans différents articles ${ }^{\mathbf{1 3}}$, d'attirer l'attention sur ce refoulement, je n'en reprendrai que les éléments nécessaires à mon propos. Ce refoulement, permet au nourrisson, en prenant appui sur le secours du recours que représente l'intervention d'un autre humain, d'obtenir un gain qui est celui de sa survie. D'une part, est jeté le voile de l'amnésie sur son infantilisme; d'autre part, la restriction de l'érogénéité à certaines parties du somatique va permettre de jeter les fondements de la première organisation sexuelle. Le refoulement organique, en ne conservant la propriété de l'érogénéité qu'à certaines parties du somatique, va permettre
13. Villa F., (2004), À propos de l'ordinaire et extraordinaire détermination humaine à rester en vie in Champ psychosomatique, $\mathrm{n}^{\circ} 35$, éd. L'esprit du temps, p.103127; (2005), La puissance de vieillir, «une façon de commencerà devenir anorganique», Psychologie clinique et projective, vol. 11, $\mathrm{p}$. 289-305; (2006), Le corps sans organe et l'organe hypocondriaque, Champ psychosomatique, 42, éd. L'esprit du temps ; (2007), Le refoulement organique et les progrès techniques de la médecine, Cliniques Méditerranéennes, 76, éd. ERES; (à paraître en 2008), Premier transfert : déssuggérer le somatique, Agora, Rio de Janeiro, Brésil. 
14. N'oublions pas que Freud nous indique dans les Trois essais surla théorie sexuelle que la zone hystérogène ne fait que révéler les modalités qui sont celles de la constitution d'une zone érogène : Freud S., (1905), Trois essais sur la théorie sexuelle, trad. $\mathrm{Ph}$. Kœppel, Paris, Gallimard, 1987, p. 82-85.

15. Freud S., [1922, (1923)], Le moi et le ça, trad. C. Baliteau, A. Bloch, J.-M Rondeau, in

Euvres complètes, psychanalyse, $t$. XVI, Paris, PUF, 1991, p. 270.

16. Freud S., (1914), Pour introduire le narcissisme, in La vie sexuelle, trad. D. Berger,

J. Laplanche et coll., Paris, PUF, 1969, p. 90.

17. Ce sont ses moments où l'on s'entend dire par un autre: «vous savez vous n'avezplus 20 ans, votre corps ne vous permet plus de faire cela». l'émergence des points de saillance qui seront les lieux de la complaisance somatique où s'accomplira la formation des zones érogènes ${ }^{14}$. Le résultat de ce mécanisme sera la possibilité nouvelle d'un dégagement de l'emprise exercée originairement par le somatique. Ces zones érogènes vont venir prendre place dans une organisation sexuelle qui se structurera sous le primat de l'une d'entre elles. Chacune des zones érogènes constituées représente un équivalent de l'organe génital dont le mode de fonctionnement sera isomorphe à celui de la zone prédominante à chaque temps de l'organisation sexuelle infantile: d'abord orale, puis anale et ensuite phallique et génitale.

$\mathrm{La}$ «désérogénéisation» de certaines parties du soma est une des exigences de la vie. Elle est le temps nécessaire pour qu'à partir d'un état somatique informe et indifférencié -lieu d'un trop d'excitabilité, émerge, par le travail psychique, un corps dont les zones érogènes constituent les coordonnées depuis lesquelles il peut être construit non seulement comme être de surface, mais aussi comme projection d'une surface ${ }^{15}$. Je ferais deux remarques. Premièrement, l'érogénéité, qui est une «activité qui consiste à envoyer dans la vie psychique des excitations qui l'excitent sexuellement ${ }^{16}$, n'a pas de coloration affective univoque, elle peut se traduire aussi bien par des sensations plaisantes que déplaisantes. En second lieu, le refoulement organique, comme tout refoulement, ne fait pas disparaître ce sur quoi il porte. Il soustrait, certes, à la conscience quelque chose qui semble, du coup, avoir disparu, avoir été abandonné ou oublié. Mais, à l'abri du refoulement, le refoulé continue d'exister, de se développer en raison de la poussée qu'il déploie pour accéder à la conscience et de l'attraction qu'il exerce sur les éléments conscients auxquels il tente de se nouer pour parvenir à son actualisation.

La perte d'érogénéité de certaines parties du soma n'est pas un phénomène irréversible et, dans certains moments psychiques de régression, ces parties peuvent retrouver cette propriété et redevenir le lieu d'une activité qui excite le psychique en le contraignant, à nouveau, au travail. Dans ces moments, qui surviennent inéluctablement, nous prenons conscience que le développement de notre corps a atteint certaines limites fonctionnelles. Dans un même temps se manifestent les premiers signes d'une involution de certaines fonctions ${ }^{17}$ et survient une configuration propice à un retour du 
refoulé organique. Nous ne pouvons plus alors douter qu'avec le temps, immanquablement, se restreint le champ des possibles - survient, alors de manière plus ou moins intense, durablement ou fugitivement, le sentiment d'assister à l'avortement de ces potentialités que nous avions crues infinies.

\section{L'INVENTION DE LA MORT PAR LE MOI}

Un jour, infailliblement, se défait l'écran faussement protecteur de la logique enfantine du " quand je serais grand, je le ferais et alors vous verrez... » qui nous a permis de supporter la nécessité pour survivre de différer la réalisation de nos souhaits et dont se soutiennent nombre de nos projets. Et quand cela survient, l'amnésie sur les débuts de notre vie psychique n'est certes pas levée, mais le poids qu'exerce sur elle ce qui en était frappé ne peut plus être méconnu. En ces instants, où nous accédons à « un peu de temps à l'état pur », surgit l'étrange rapport que l'homme entretient avec la mort. Le moi est contraint de se rendre à l'évidence de la finitude de l'être humain, de son essentielle faiblesse et fragilité face au tellurisme pulsionnel. D'une part, il lui faut envisager l'œuvre de ce qui, jour après jour, participe à la décomposition de la forme et du visage humains. Et, d'autre part, mais dans le même temps, apparaît, tragiquement, que, quelle que soit la force de la pulsion de vie, elle ne sera pas en mesure de continuer éternellement à recréer, par son travail de liaison, des arrangements, des formes, des organisations du moi, qui pourront se substituer à ceux que la déliaison a déconstruits, décomposés ou détruits. Plus exactement, elle ne sera plus à même de lier les représentations d'une manière telle que «je» puisse continuer à me reconnaître en ayant l'illusion d'une continuité de soi et le sentiment de la préservation d'une identité mö̈que.

Le moi, en prenant, dans cet instant fugitif, la mesure de tout l'insatisfait qui, avec le temps, s'est accumulé au cœur de l'humain, est, certes, confronté à tout ce qui n'a pas encore été traité psychiquement, mais surtout à un noyau de choses qui semblent s'avérer intraitables pour et par le psychique et qui, pourtant, constitue le noyau palpitant de l'être. Alors, la mort, dont nous ne parvenions pas, malgré les morts qui ont pu survenir autour de nous, à réellement croire qu'elle est notre 
18. «Brefl'artiste, le

Temps, avait "rendu" tous ces modèles de telle façon qu'ils étaient reconnaissables; mais ils n'étaient pas ressemblants, non parce qu'ils les avaitflattés, mais parce qu'il les avait vieillis. Cet artiste-là, du reste, travaille fort lentement », Proust M., ibid., p. 936. perspective certaine et dont il semblerait bien que nulle représentation ne soit présente dans l'inconscient, nous apparaît comme un horizon dont nous devons percevoir toute la duplicité. Non seulement, elle est cette issue fatale et redoutée qu'avec succès, nous avions pu feindre, jusque-là, d'ignorer, mais, ultime ruse de la raison, elle peut devenir désirable pour et par le moi qui peut «l'accepter» et la «choisir» plutôt que de se voir soumis inéluctablement à une dérive encore plus grande de son identité. C'est en ce sens que l'on pourrait avancer que le moi invente la mort comme solution pour ne pas avoir à assister à sa destruction par la pulsion. Le moi se donne ou accepte la mort pour sauver l'intégrité qu'il lui reste.

\section{LE DÉSHABILLAGE DU MOI: ALLER AU-DELÀ DE «NOTRE» CORPS VERS LE RÉEL OÙ IL PREND SOURCE}

Mais, nous n'en sommes pas encore rendu à cette nécessité-là, juste à celle où l'on commence à ne plus pouvoir croire que l'on se ressemble ${ }^{18}$ suffisamment pour faire fi de l'œuvre et des injures du temps. L'instant du souvenir involontaire provoque un déshabillage du moi. C'est un moment douloureux où se lève partiellement le rétrécissement de la perception qui nous permettait de voir le monde chaussé des lunettes de notre réalité psychique : c'est à dire à l'aune de ce principe de réalité, qui n'est rien de plus qu'une modification réaliste du principe de plaisir. Cet instant nous conduit, au-delà de ces deux principes, vers un réel où prend son assise ce que nous reconnaissons comme «notre » corps. Sa fonction étant d'être un écran placé entre ce réel et le psychique qui, habituellement, cache celui-là à celui-ci, en lui permettant néanmoins de se former en tant qu'image perceptible sur la surface de projection qu'il constitue. Car, il ne faut pas oublier que ce qui est visible ou plutôt perceptible par le psychique en tant que corps n'est que la partie exondée d'un immense territoire inconnu, méconnu qui relève de la dimension de l'invisible, voire de l'invisibilité. Ce territoire est, à notre opinion, celui que Freud désigne du nom de somatique.

Mais, revenons au déshabillage, il est de même nature que celui auquel nous procédons chaque nuit en nous mettant au lit pour dormir. Voici comment Freud, avec humour, le décrit : 
«Nous ne sommes pas habitués à attacher beaucoup de pensées au fait que l'homme, chaque nuit, dépose les enveloppes dont il a revêtu sa peau, et aussi, éventuellement, les pièces complémentaires de ses organes corporels, dans la mesure où il a réussi à en couvrir les manques par un substitut tel que les lunettes, faux cheveux, fausses dents, etc. Il est permis d'ajouter qu'en allant dormir, il procède à un déshabillage tout à fait analogue de son psychique, renonce à la plupart de ses acquisitions psychiques, et ainsi des deux côtés il instaure un extraordinaire rapprochement avec la situation qui fut le départ du développement de sa vie »19.

La différence entre les deux moments de déshabillage réside dans le fait que ce que nous faisons avant le coucher, sans y prendre habituellement garde, requiert cette fois-ci instamment notre attention. Le déshabillage capte celle-ci au point de s'imposer à notre perception. C'est un moment métaphysique où la vanité de tout notre art de la prothèse palliative est ressentie et où, pour un instant, nous cessons d'être dupes des artifices ingénieux dont nous avons usés pour nous cacher que nous n'avions surmonté que pour partie notre infantilisme face aux exigences de la vie pulsionnelle. C'est aussi un moment de vérité où nous percevons la précarité notre être, la nature fondamentalement instable de la vie et la finitude matérielle de notre existence. Et c'est, enfin, un moment déséquilibré d'incertitude où nous saisit l'interrogation sur le sens et la fonction de ce que nous avons fait, de ce que nous faisons et de ce que nous ferons au regard de ce qui profondément nous anime et nous meut pulsionnellement.

Lorsque nous nous souvenons, d'une part, que le moi est avant tout un moi corporel ${ }^{20}$ et, d'autre part, que «les mêmes organes et systèmes d'organe sont d'une manière générale à la disposition des pulsions sexuelles tout comme des pulsions $d u$ moi ${ }^{\mathbf{2 1}}$, nous ne pouvons plus être surpris que le corps soit l'un des lieux privilégiés par où survient l'ébranlement affectif de l'être. Le moment de déshabillage est celui d'un désinvestissement libidinal de chacune de ces enveloppes, de ces prothèses et de ces acquisitions dont nous avions parés notre originel dénuement pour en oublier la crudité cruelle (cela s'inscrit dans le passage culturel du cru au cuit). Ce mouvement de déliaison pulsionnel défait l'organisation psychique construite sous le primat et sur le modèle d'une zone érogène prédominante. D'une part, chacune des parties de l'ensemble
19. Freud S. [1915, (1917)], Complément métapsychologique à la doctrine du rêve », trad. J. Altounian, A.

Bourguignon, P. Cotet, A. Rauzy, in Euvres complètes, psychanalyse, $t$. XIII, Paris, PUF, 1988, p. 246.

20. Freud S., [1922, (1923)], Le moi et le ça, op. cit., p. 270

21. Freud S. (1910), Le trouble de vision psychogène dans la conception psychanalytique, trad.. P. Cotet, in Euvres complètes, psychanalyse, $t$. X, Paris, PUF, 1993, p. 183. 
précédemment constitué est rendue à sa logique partielle et partiale propre et, d'autre part, émergent d'autres parties du soma qui avaient été repoussées sous l'ensemble en tant que fondement pouvant être négligé et qui retrouvent leur érogénéité.

La déconstruction de l'ensemble comme organisation lui fait perdre sa fonction d'écran et nous prive de la force de contre-investissement que nous pouvions déployer à partir d'elle pour maintenir les refoulements organiques originaire et secondaires. En relâchant l'investissement libidinal d'une zone érogène, c'est sa représentation psychique qui vacille. Dans l'intervalle de cette vacillation, se révèle derrière la fonction érotique que l'organe avait prise, sa fonction originairement non psychique. C'est alors aussi que devient, fugacement, perceptible l'œuvre du traitement psychique du pulsionnel et le travail psychique de «désuggestion » par lequel nous avons pu nous arracher à une emprise trop grande du somatique. Pour suivre ce que nous proposons, nous devons rappeler jusqu'où va le raisonnement de Freud dans son article sur «Le trouble de vision psychogène dans la conception psychanalytique». Il avance que lorsque le moi perd la domination sur un organe qui s'est vu mettre entièrement à la disposition de la pulsion sexuelle refoulée, il peut jeter l'enfant avec l'eau du bain et il peut pousser l'œuvre du refoulement jusqu'à, dans l'exemple étudié, fonctionner comme si l'œil n'existait plus pour lui. Il ne veut rien voir par cet organe où les intérêts sexuels sont devenus prédominants ${ }^{22}$.

\section{DES OCCASIONS PROPICES À L'ÉBRANLEMENT AFFECTIF}

L'occasion de cet ébranlement affectif peut être quelconque et fort varié, sa survenue, inéluctable, ne peut pourtant pas être anticipée, nous ne disposons d'aucun moyen pour cela et sommes à la merci des contingences. Mais, il nous semble certain que la douleur, la parole, le regard de l'autre et la perception, le contact du corps d'autrui forment des occasions particulièrement propices pour cet élargissement non-désiré de notre perception de l'évolution de notre corps et, donc, de notre être. Ce qui provoque un trouble dans la perception du développement de notre corps, que nous souhaiterions linéaire 
et ascendant, ce sont les modifications normales ou pathologiques qui, survenant dans le cours des vicissitudes de l'existence, atteignent et affectent le corps : les maladies et les limitations fonctionnelles qui accompagnent notre vieillissement, bien sûr, mais aussi les changements qui sont liés à notre relation à autrui et qui agissent sur la vie affective et amoureuse.

Nous pouvons difficilement ignorer le rôle primordial que joue la douleur dont "la façon dont on arrive d'une manière générale à la représentation de son corps propre $»^{23}$. Nous ne savons que trop, comment, à l'occasion d'une affection somatique douloureuse, soit nous acquérons une nouvelle connaissance de ou des organe(s) touché(s), soit nous prenons, pour la première fois, conscience de l'existence de cette partie du corps qui était restée hors de toute représentation jusque-là. En vieillissant, il est de plus en plus fréquent que, sans aller jusqu'à la douleur, nous ressentions le poids de la matérialité du corps et que nous éprouvions, en raison de cela même, des préoccupations hypocondriaques. Nous éprouvons des gênes physiques, nous ressentons les effets de la perte de certaines souplesses corporelles et/ou psychiques, en un mot nous avons affaire à ce que signifie être chargé d'ans. Ce sont autant d'occasions qui surviennent au détour de l'une de nos entreprises quotidiennes, à la faveur d'un souvenir que convoque la situation présente. Ce sont là des moments où nous réalisons ce que notre corps est devenu, ce qu'il est en train de devenir et où, dans la défaillance complète ou partielle d'une fonction ou d'un organe, nous prenons conscience de son existence que son bon fonctionnement nous avait jusque-là rendu imperceptible.

Ce sont des moments où ne pouvons plus complètement méconnaître que la mort travaille, tout le temps, dans le vivant. Son œuvre de destruction n'est, certes, pas paradoxalement seulement au service de la mort et s'avère même être une condition de possibilité pour la vie. La mort de certaines parties du vivant est exigée pour que soit assurée la viabilité et la survie de l'ensemble dont elles font parties (dans des circonstances dites normales, lorsque qu'un organe a atteint un certain développement, les cellules spécifiques qui le constituent perdent momentanément ou régulent leur fertilité pour qu'il ne se développe pas au-delà d'une limite qui représenterait un danger létal pour l'organisme - mais, nous ne connais-
23. Freud S., [1922, (1923)], Le moi et le ça, op. cit., p. 270. 
sons aussi que trop les cas qui font exception à cette règle).

La mort, nous l'avons rappelé plus haut, sculpte le vivant. Mais, elle n'est pas aussi sans le détruire radicalement, car dans la course conflictuelle que se livrent agents de mort et agents de vie dans l'organisme, le rapport de force bascule, tôt ou tard, du côté des agents de mort qui semblent disposer des plus forts bataillons. Le pouvoir de renouvellement et de régénération dont disposent les forces de vie n'est pas illimité. Penons quelques exemples. Les cellules qui composent la peau, disposent, avec le temps, de moins en moins de la capacité de remplacer les cellules mortes par de nouvelles : la peau perd de son élasticité, les rides, comme autant de marques de l'œuvre de la mort, creusent le corps, la tendance à l'embonpoint gagne du terrain, la cellulite impose son indésirable présence. Dans le même temps, les effets ostéoporotiques rendent notre ossature plus fragile et, sans en être toujours perclus, nous sommes plus enclins au rhumatisme, à l'arthrite, à l'arthrose. Et n'oublions pas, pour clore cet aguichant tableau, les risques cardiovasculaires, dont nous avions tant été prévenus, sans y croire, qui deviennent réalité. Ce sont là des sollicitations pour le psychique qui se font de plus en plus fréquentes et dont l'insistance contraint à des remaniements qui traduisent que, tant bien que mal, plus ou moins à contrecœur, nous réalisons psychiquement pour partie ce qui nous arrive.

\section{LES MULTIPLES PERTES DE L'OBJET MOI}

Dans ce qui arrive, il nous faut distinguer une perte d'objet particulière et singulièrement déstabilisante. Le moi est l'un des premiers objets de la libido - le premier peut-être même. Il est en tout cas cet objet singulier qui va se construire en devenant le lieu où se concentre toute la libido au point de pouvoir être pensé par Freud comme son réservoir. Il est le lieu d'où, par cession partielle et momentanée d'une part de la libido, d'autres objets que le moi pourront être investis en dehors du moi, dans le monde. Malgré sa prétention à l'unité et à la constance, le moi n'est qu'une instance de synthèse toute relative dont la forme est fondamentalement instable et bien plus sujette aux modifications et aux altérations que le moi ne peut ni le concevoir, ni l'imaginer, ni surtout l'admettre. 
Derrière l'organisation présente du moi qui est le lieu de l'actuel investissement libidinal, il nous faut concevoir l'innombrable série de tous les «moi » qui ont antérieurement existés et prédominés et dont la fonction moi a dû, pour perdurer, accepter l'altération, la modification, voire la destruction.

À chacune de ces métamorphoses, a eu lieu nécessairement un désinvestissement libidinal de l'ancien moi au profit du nouveau. Il est difficile de penser que ces remaniements se font facilement, sans peine. Probablement, à chaque fois que se produit une perte de l'objet moi, surgit la douleur - réaction propre à la perte d'un objet. Celle-ci durera tant que ne sera pas effectué le deuil de cette forme antérieure du moi, c'est d'ailleurs la condition pour qu'un nouveau moi puisse être investi libidinalement. Ces expériences de perte du moi, qui se sont répétées un nombre de fois incalculables et dont la mémoire s'empresse d'effacer, autant que possible, les souvenirs pénibles qui s'y rattachent, ont pour effet d'accroître en nous une vigilance pour que ne se reproduise plus une telle situation. Notre propension à l'angoisse trouve, là, un terrain d'élection pour se manifester comme signal d'un danger qui pourrait entraîner la perte du moi.

L'indésirable répétition de la perception des modifications que le temps impose au corps, et la difficulté dans laquelle nous sommes de ne plus pouvoir continuer à les ignorer, a pour effet de nous obliger à prendre acte que notre représentation $\mathrm{du}$ corps est devenue caduque et que nous devons remanier notre image du moi-corps. Mais, dans l'intervalle entre ces deux images : l'ancienne et celle qui devrait advenir, survient une régression où est réactivée l'expérience dite du stade du miroir. Nous sommes contraints, par nécessité, de nous détacher, de nous désolidariser de l'image qui prédominait dans notre représentation. En perdant ce point de repère, nous revoyons, au-delà du miroir, s'éloigner encore plus la perspective d'atteindre à nouveau, plus tard, l'Urbild, la forme originaire du moi que, dans un moment de jubilation, le nourrisson avait pris pour sa réalité. Une fois encore, l'humain prend la mesure de ce qui lui manque pour être à la hauteur des exigences, non seulement, de la vie mais, aussi, de son idéal. Mais, avec le temps, l'homme doit admettre de plus en plus, comme un réel incontournable et foncièrement insupportable, que le temps ne travaille pas pour lui et que le manque, loin de 
24. Freud S. (1910), Le trouble de vision psychogène..., op.cit., p. 186.

25. Pour mémoire le texte sur Le trouble de vision... est de 1910, celui de Pour introduire le narcissisme où Freud propose sa définition de

l'érogénéité comme étant une propriété générale de tous les organes est de 19131914. Ce dernier texte, trad. par J. Laplanche, dans Freud S., Euvres complètes, psychanalyse, $t$. XII, Paris, PUF, 2005, plus particulièrement, p. 227-228.

26. Freud S. (1910), Le trouble de vision psychogène..., op. cit., p. 185-186.

27. Groddeck G., Ça et moi, trad. R. Lewinter, Paris, Gallimard, 1977, p. 44. diminuer, ne va cesser de s'accroître. Cela se manifeste, entre autres, dans l'accumulation des défaillances, de plus en plus fréquentes, du corps qui le font s'éloigner encore plus de l'idéal qu'il espérait atteindre un jour.

Nous reprendrons maintenant la question que Freud nous pose dans «Le trouble de vision psychogène dans la conception psychanalytique»: "On peut se demander si la répression des pulsions partielles sexuelles engendrée par les influences de la vie suffit à elle seule à provoquer les troubles fonctionnels des organes ou s'il ne faut pas que soient présentes des conditions constitutionnelles particulière, qui d'emblée amènent les organes à l'exagération de leur rôle érogène et provoquent par là le refoulement des pulsions $»^{24}$.

Nous répondrons positivement cette question. Oui, il faut que soient présentes des conditions constitutionnelles particulières et nous proposerons de reconnaître, dans la capacité dont dispose tout le soma d'être érogène, l'une de ces conditions ${ }^{25}$. À l'occasion des perceptions des modifications du corps, ce qui se produit est une ré-érogénéisation de zones du corps auxquelles le refoulement organique avait fait perdre manifestement cette propriété. Elles redeviennent, pour le meilleur et pour le pire, le lieu d'une "activité qui consiste à envoyer dans la vie d'âme des stimuli qui l'excitent sexuellement » et qui, parce que "l'animique repose sur l'organique », constitue une exigence de travail pour le psychique. Cette articulation étroite entre le somatique et le psychique, Freud pense que la psychanalyse, en allant à l'encontre de notre propension naturelle, contraint à ne jamais l'oublier. Mais, posant cela, il ne méconnaît pas que le psychanalyste devra convenir que le travail psychanalytique ne peut poursuivre l'œuvre du psychique que jusqu'à ce fondement, sans pouvoir aller au delà et sans pouvoir abolir la caractère énigmatique de leur connexion ${ }^{26}$. Freud avancera une piste de réflexion supplémentaire dans une lettre du 5 juin 1917 à Groddeck : l'inconscient est peut être le missing link entre psyché et soma ${ }^{27}$.

\section{LA RELATION À AUTRUI}

Notre réflexion ne peut pas négliger les effets d'ébranlement affectif qui résultent de notre rapport à autrui. Nous savons depuis le commentaire de Winnicott que la mère, la 
famille, puis, plus tard, dans certaines circonstances, d'autres humains jouent pleinement le rôle du miroir dans le développement de l'enfant puis, de l'adulte ${ }^{28}$. Winnicott et Lacan, sans ignorer les effets pathologiques des ratés qui surviennent à ce stade, ont étudié la fonction structurante, constructrice jouée par autrui. Nous porterons notre attention sur les moments ultérieurs de reprise du stade du miroir où se produisent des déstructurations, voire des destructions de la fonction originaire. Dans les meilleurs des cas, déstructuration et destruction ne sont pas durables, elles ont un caractère passager à la suite duquel advient une nouvelle structuration. Ces reconstructions sont permises par l'aptitude à la synthèse du moi. La perception, au-delà du miroir, que représente autrui pour nous, peut, comme nous l'avons évoqué précédemment, nous renvoyer une image de nous même présentant une telle discordance avec l'image de soi-même qu'en résulte un trouble identitaire plus ou moins aigu. Cela dépend probablement de l'intensité libidinale de la fixation qui nous lie à cette image.

Mais, c'est un autre point de la relation à autrui que nous voudrions examiner. Nous savons que la construction de notre vie psychosexuelle a dû, en raison de notre infantilisme, traverser différents moments d'organisation sexuelle. Chacun d'eux s'est vu placé sous le primat d'une zone érogène qui, dans tous les cas, tenait lieu d'un équivalent d'organe génital. En raison des nécessités de la vie, nous avons dû abandonner successivement les organisations sexuelles prégénitales et leur mode spécifique de satisfaction. Cela s'est effectué au profit de la prédominance d'une organisation génitale qui, pour la satisfaction, impose le passage par un autre que soi-même et permet ainsi que l'individu accomplisse la fonction reproductrice. Or, voici, qu'après s'être plié, au moins partiellement, à ces abandons successifs et être parvenu à supporter les pertes que cela imposait ${ }^{29}$, l'individu voit l'activité de l'érogénéité non seulement reprendre de la vigueur dans certaines de ces zones abandonnées, mais se manifester dans d'autres qu'il ne savait pas pouvoir être le lieu d'un tel dynamisme.

Et, cette expérience de vie survient au moment même où, pour l'un des deux sexes, est perdue la capacité de donner la vie. L'une des raisons de s'être plié au primat du génital était liée justement à l'étrange et implacable désir de reproduction qui semble habiter, au-delà de toute raison, la plupart des humains. L'événement de la ménopause, a indéniablement des
28. Winnicott D. W., (1967), Le rôle de miroir de la mère et de la familled ans le développement de l'enfant, trad. C. Monod et J.-B. Pontalis, in Jeu et réalité, Paris, Gallimard, 1971, p. 153-162.

29. C'est, selon nous, le refoulement organique secondaire qui rend possible l'abandon partiel des zones érogènes antérieurement surinvesties. 
30. Je renvoie à mon article:(2006), Le corps sansorgane et l'organe hypocondriaque, Champ psychosomatique, 42, éd. L'esprit du temps. effets sur le partenaire de la femme. Au risque de paraître schématique, je n'hésiterai pas à dire qu'enfant, nous nous étions résignés tant bien que mal, à admettre que nos parents avaient dû certes faire l'amour, mais ce n'était ni par désir, ni par envie, juste pour avoir des enfants et surtout pour nous avoir nous. En grandissant, nous avons eu à surmonter notre peur de l'autre sexe et notre dégoût de la chose sexuelle. Ce n'est certes pas seulement pour avoir des enfants que nous y sommes parvenus, tant bien que mal, plus ou moins effectivement, mais cette éventualité n'était pas sans nous tracer, plus que nous ne l'admettrons jamais, une voie constitutionnelle que la culture n'a fait que renforcer. Quand disparaît la raison reproductrice qui contribua à nous faire accepter de passer sous le joug d'un relatif primat génital, font retour les différentes formes de la sexualité infantile qui avaient été refoulées sous les exigences de la sexualité dite adulte.

La résurgence de l'érogénéité dans nombre de parties du corps d'où elle avait été refoulée vient ébranler encore plus ce primat, déjà bien fragile, du génital. Il nous faut alors nous affronter au dégoût que nous ressentons, croyons-nous, pour ce corps vieillissant, alors qu'il n'est principalement que celui que nous éprouvons pour ce corps qui s'anime d'une activité et d'une exigence que le moi ne souhaite pas prendre en considération. Ce que nous appelons les faiblesses de la chair ne sont que l'expression de la force des pulsions qui œuvrent dans tout le corps, gouvernant aussi bien la vie psychique que la vie végétative. Notons que ce qu'on appelle les faiblesses de la chair n'a pas pour synonyme les faiblesses $d u$ corps ; cellesci révéleraient plutôt notre incapacité à répondre des et aux faiblesses de la chair. La mise en relation de ces deux expressions nous permet de faire entendre que la chair est un au-delà du corps psychique, un lieu qui excède toute image du corps, un espace où se défait l'organisation du corps à partir des organes identifiés qui le composent ${ }^{30}$. La chair est le lieu d'une animation qui, se révélant intraitable, peut mettre en danger l'existence de la vie psychique. 


\section{L'EXCITATION SEXUELLE ET LA LIBIDO, L'INSUFFISANCE DE LA PSYCHÉ}

Notre corps, au contact du corps de l'autre, est particulièrement confronté au retour de ces formes de sexualité que nous croyions révolues et à l'apparition d'un champ de possibles qui excèdent ce que peut accueillir la psyché. C'est, par la voie, ici, indiquée que nous parvenons à donner un sens à ce que Freud avançait en 1895 lorsqu'il tentait de cerner les conditions étiologiques de la névrose d'angoisse en examinant les différentes circonstances prédisposantes : «L'angoisse dans le senium (climatère des hommes) requiert une autre explication. Ici, la libido ne faiblit pas; mais il se produit, comme pendant le climatère des femmes, un tel accroissement dans la production de l'excitation somatique que la psyché s'avère insuffisante, de façon relative, à maîtriser cette dernière ». Et plus loin, il poursuit: "Le cas du veuvage et celui de l'abstinence voulue, de même que le cas du climatère, se règlent chez la femme sans doute de la même façon que chez l'homme, mais pour le cas de l'abstinence s'ajoute certainement encore le refoulement intentionnel du cercle de représentations sexuel, refoulement auquel la femme abstinente, luttant avec la tentation, doit se décider fréquemment, et il est possible qu'à l'époque de la ménopause l'horreur que la femme vieillissante ressent à l'égard de la libido devenue excessive agisse de façon semblable $»^{31}$.

La difficulté à laquelle nous confronte le vieillissement n'est pas, comme nous pourrions le croire hâtivement, liée à une baisse soit de l'excitation sexuelle somatique, soit de la libido, mais au contraire à l'accroissement dans tous les cas de l'une et/ou de l'autre ${ }^{32}$. La faiblesse qui est, ici, en cause est celle de la psyché qui, avec le temps, s'avère insuffisante à maitriser ce surcroît d'excitation.

Nous avons évoqué le rôle d'autrui comme miroir, mais nous ne pouvons pas méconnaître que, parfois, il devient le lieu où nous percevons, au-delà de son corps, la crudité du réel de la chair dont l'un de nos premiers mouvements serait de nous détourner. Sur le visage et sur le corps des proches, de l'être aimé, nous voyons se profiler le visage de la mort. Cela survient un peu à la manière dont Giacometti parvient à arracher le portrait qu'il fait de son modèle à la passion de la
31. Freud S., [1894, (1895)], Du bien-fondé à séparer de la neurasthénie un complexe de symptômes déterminé en tant que « névrose d'angoisse », trad. J. Stude-Cadiot, Fr.-M. Gathelier, in Euvres complètes, psychanalyse, t. III, Paris, PUF, 1989, p. 52-53, (je souligne)

32. Cliniquement, il est important de distinguer les effets de l'augmentation de l'excitation somatique, de celle de la libido et il ne faut pas négliger les conséquences de l'intrication des deux excitations. 
33. Baudelaire C., Les Fleurs du mal, in Euvres complètes, Paris, La Pléiade, Gallimard, 1975, p. 31-32. ressemblance réaliste et à faire émerger des gribouillis, qu'il a jetés sur la toile, le tourbillonnement brownien par lequel apparaît que tout visage et tout corps est un lieu d'activités pulsionnelles. Dans ses portraits, se laisse voir, de manière vive, la mort à l'œuvre et, dans le visage du vivant, se dessine le squelette qu'un jour, devenu cadavre livré à la décomposition, il sera. Vieillir avec l'un de ses contemporains nous expose à vivre ce que chante cruellement et tragiquement le poème de Baudelaire intitulé «Une charogne »33. Se promenant, ce beau matin d'été si doux, avec son âme, son amante aimée, ils rencontrent une charogne infâme, les jambes en l'air, brulantes et suants les poisons, le ventre plein d'exhalaison. Cette pourriture est une carcasse prête pourtant à s'épanouir comme une fleur. "On eût dit que le corps, enflé d'un souffle vague / Vivait en se multipliant ». De ce terrible spectacle, le poète ne se détourne que pour regarder vers sa belle et supporter d'avoir à penser :

«-Et pourtant vous serez semblable à cette ordure

À cette horrible infection,

Etoile de mes yeux, soleil de ma nature,

Vous, mon ange, ma passion!

Oui, telle vous serez, ô la reine des grâces,

Après les derniers sacrements,

Quand vous irez, sous l'herbe et les floraisons grasses

Moisir parmi les ossements.

Alors, ô ma beauté! dites à la vermine

Qui vous mangera de baisers,

Que j'ai gardé la forme et l'essence divine

De mes amours décomposés!».

Certes, me direz-vous, il est plus facile d'avoir à penser la mort de l'autre et sa décomposition que la sienne propre. Nous ne fonctionnons que, trop aisément, sur le modèle du trait d'esprit que Freud rapporte. L'un des partenaire d'un couple dit à l'autre: «Lorsque que l'un de nous deux mourra, j'irai à Paris ». Il n'en demeure pas moins que le poète nous désigne, ici, la force d'un lien qui s'oppose au travail de la décomposition et le supporte. Il ne se contente pas de parler de la décomposition de l'autre aimé, mais de l'inévitable décomposition de nos amours et de nous-mêmes. Nous reste alors les 


\section{LA CHAIR DU PSYCHIQUE}

ressources que trouverons dans notre capacité à garder la forme et l'essence de ce qui nous unit à cet autrui-là.

\section{UNE CONFESSION POUR CONCLURE}

Avant de clore ce texte, je confesserai une gêne technique que j'ai éprouvé tout au long de sa rédaction. Son écriture m'a confronté à la réalité qu'il m'était plus facile d'écrire sur le vieillissement quand je pouvais croire, malgré mes affirmations, que cela ne me concernait qu'indirectement, qu' aujourd'hui où j'ai indéniablement vieilli. Je vais donc arrêter mon propos. Et, jusqu'à l'un de ces prochains instants, qui ne sauraient tarder, où j'accéderai, fortuitement à un peu de temps à l'état pur, je vais m'illusionner dans le sentiment préservé jalousement que, bien que le temps ait passé, je me sens toujours aussi jeune qu'à l'orée de ma vie et que me reste une infinité de bons et de mauvais jours avant que moi ne concède que la partie est inévitablement perdue. Je replonge, donc, dans la vie qui, le plus souvent, est «une perpétuelle distraction qui ne nous laisse même pas prendre conscience de ce dont elle distrait ${ }^{\mathbf{3 4}}$.

\section{BIBLIOGRAPHIE}

AMEISEN J.-C., La sculpture du vivant, Paris, Points Sciences, Seuil, 2003. BAUDELAIRE C., Les Fleurs du mal, in CEuvres complètes, Paris, La Pléiade, Gallimard, 1975.

BENJAMIN W., Pour le portrait de Proust, in Mythe et violence, trad. M. de Gandillac, Paris, Denoël.

FÉDIDA P. (1985), Restes diurnes. Restes de vie in Crise et contre-transfert, Paris, PUF, 1992.

FERENCZI S. [(1920-1922) publication posthume (1934)], «Réflexions sur le traumatisme », trad. Coq Héron, in CEuvres complètes, Psychanalyse 4, Paris, Payot, 1982.

FREUD S. [1894, (1895)], Du bien-fondé à séparer de la neurasthénie un complexe de symptômes déterminé en tant que «névrose d'angoisse », trad. J. Stude-Cadiot, Fr.-M. Gathelier, in Euvres complètes, psychanalyse, t. III, Paris, PUF, 1989.

FREUD S., [1901, (1905)], Fragments d'une analyse d'hystérie, trad. F. Kahn, F. Robert, in CEuvres complètes, psychanalyse, $t$. VI, Paris, PUF,
34. Kafka F, Préparatifs de noces à la campagne, Gallimard, L'imaginaire, éd de 1988, p. 378. 
2006.

FREUD S., (1905), Trois essais sur la théorie sexuelle, trad.. Ph. Koppel, Paris, Gallimard, 1987.

FREUD S. (1910), Le trouble de vision psychogène dans la conception psychanalytique, trad. P. Cotet, in Cuvres complètes, psychanalyse, $t . X$, Paris, PUF, 1993.

FREUD S., (1914), Pour introduire le narcissisme, in La vie sexuelle, trad. D. Berger, J. Laplanche et coll., Paris, PUF, 1969.

FREUD S. (1914), Pour introduire le narcissisme, trad. J. Laplanche, in Euvres complètes, psychanalyse, $t$. XII, Paris, PUF, 2005

FREUD S. [1915, (1917)], Complément métapsychologique à la doctrine du rêve, trad. J. Altounian, A. Bourguignon, P. Cotet, A. Rauzy, in CEuvres complètes, psychanalyse, $t$. XIII, Paris, PUF, 1988.

FREUD S., [1922, (1923)], Le Moi et le Ça, trad. C. Baliteau, A. Bloch, J.-M Rondeau, in Euvres complètes, psychanalyse, t. XVI, Paris, PUF, 1991.

FREUD S., [1925, (1926)], Inhibition, symptôme et angoisse, trad.. J. Doron, R. Doron, in Euvres complètes, psychanalyse, t. XVII, Paris, PUF, 1992.

FREUD S., Résultats, idées, problèmes, trad. J. Altounian, A. Bourguignon, P. Cotet, A. Rauzy, in Résultats, idées, problèmes, t. II, Paris, Gallimard, 1985.

GRODDECK G., Ça et moi, trad. R. Lewinter, Paris, Gallimard, 1977

JOYCE J., Ulysse, trad.. A. Morel, Paris, livre de poche, 1965.

KAFKA F., Préparatifs de noces à la campagne, Paris, Gallimard, 1988.

PROUST, M., A la recherche du temps perdu, t. III, Paris, La Pléiade, Gallimard, 1977.

VILLA F. (1989): Les étrangers du jour dans l'intimité de la nuit, Nouvelle Revue de Psychanalyse, 40, Paris, Gallimard, pp. 127-146.

VILLA F. (2004), À propos de l'ordinaire et extraordinaire détermination humaine à rester en vie, Champ psychosomatique, pp.103-127.

VILLA F. (2005), La puissance de vieillir, « une façon de commencer à devenir anorganique », Psychologie clinique et projective, 11, pp. 289305.

VILLA F. (2006), Le corps sans organe et l'organe hypocondriaque, Champ psychosomatique, 42 , pp. 33-46.

VILLA F. (2007), Le refoulement organique et les progrès techniques de la médecine, Cliniques Méditerranéennes, 76, pp. 45-60.

VILLA F. (2007), Les névroses mixtes: une leçon de méthode nosographique, L'évolution psychiatrique, 72-3, pp. 477-487.

VILLA F., (à paraître en 2008), Premier transfert : déssuggérer le somatique, Agora, Rio de Janeiro, Brésil.

WINNICOTT D. W. (1967), Le rôle de miroir de la mère et de la famille dans le développement de l'enfant in Jeu et réalité, trad. C. Monod et J.-B. Pontalis, Paris, Gallimard, 1971 


\section{RÉSUMÉ}

Il est des instants de souvenir fortuit où advient « un peu de temps à l'état pur $»$. Il se produit alors un ébranlement affectif où survient le temps de l'affect. Ce sont des moments de saillance où le somatique va à la rencontre du psychique. Ce sont des moments où s'impose le déshabillage du moi et où l'individu va au-delà de «son» corps vers le réel où il prend source. La douleur, le regard et la parole de l'autre, la perception et le contact du corps d'autrui sont autant d'occasions propices à cet ébranlement affectif qui se traduit par une levée du refoulement organique. En ces circonstances, le moi fait l'expérience d'une perte du moi comme objet libidinal et il se voit contraint à un travail de deuil dont l'issue permettra ou non la construction d'un nouveau moi investi libidinalement. La réalité de la relation à autrui, dans ce cheminement, est déterminée par la capacité à envisager l'œuvre de la mort dans le vivant et à ne pas reculer devant la décomposition inéluctable du corps. Des remarques sur le travail de la pulsion de mort conduisent à l'hypothèse d'une invention de la mort par le moi et à ne pas négliger de tenir compte, dans la clinique, de l'insuffisance de la psyché face à l'excitation somatique et à la libido.

Mots-clés : Âge - Corps - Douleur - Mort - Refoulement organique Refoulement - Soma - Sexuel - Temps - Vieillissement.

\section{SUMMARY}

There are moments of chance memory which bring with them moments of "pure time". An affective shock occurs, with the appearance of the time of the affect. These are moments of salience when the somatic meets the psychic; times when the undressing of the ego becomes necessary and the individual goes beyond "his own" body towards the real, from which it takes its source. Pain, the attitude and the words of the other, the perception of and contact with the body of the other are all conducive to this affective shock, which takes the form of the abolition of organic repression. In these circumstances, the ego experiences the loss of the ego as a libidinal object, and is obliged to go through a mourning experience at the end of which a new ego, invested libidinally, will or will not be constructed. In this progression, the reality of the relationship with the other is measured by the ability to contemplate the workings of death in the living, and not to be repelled by the inevitable decomposition of the body. Remarks on the work of the death impulse lead to the hypothesis of an invention of death by the ego, and active consideration, in clinical analysis, of the inadequacy of the psyche in the face of somatic excitement and libido.

Key-words : Age - Body - Pain - Death - Organic repression - Repression - Soma - Sexual - Time - Ageing. 